Universidad Católica del Norte

Antofagasta - Chile

\title{
ON A VARIATIONAL INEQUALITY FOR A HYPERBOLIC-PARABOLIC EQUATION WITH A LIPSCHITZIAN NONLINEARITY
}

\author{
Jorge Ferreira* \\ Universidade Estadual de Maringá, Maringá-Brazil
}

\begin{abstract}
In this paper we prove the existence and uniqueness of global weak solutions to the mixed problem for the degenerate nonlinear hyperbolicparabolic inequality$$
\text { (*) }\left\{\begin{array}{c}
K_{1}(x) u^{\prime \prime}+K_{2}(x) u^{\prime}-\Delta u+F(u) \geq f \text { in } Q=\Omega \times(0, T), \\
u^{\prime} \geq 0 \text { in } Q, \\
u=0 \text { at } \sum=\Gamma \times(0, T), \\
u(x, 0)=u_{0}(x), u^{\prime}(x, 0)=u_{1}(x), x \in \Omega,
\end{array}\right.
$$

where $Q$ is a cylindrical domain of $R^{n+1}$ with the lateral boundary $\sum, \Omega$ is a bounded domain of $R^{n}$ with smooth boundary $\Gamma, F(s)$ is a Lipschitzian real function such that $s F(s) \geq 0, \forall s \in R$. Here $K_{1}(x)$ and $K_{2}(x)$ are real functions defined on $\Omega$ such that $K_{1}(x) \geq 0$ and $K_{2}(x)>0$ a.e on $\Omega$, which satisfy some appropriate conditions and $\Delta=\sum_{i=1}^{n} \frac{\partial^{2}}{\partial x_{i}^{2}}$. For the existence we apply the penalty operator method (see Lions [12]), the Galerkin's approximation method plus compactness argument. The uniqueness is considered only for some particular case of $F$ which permit the application of a method due to Visik and Ladyzenskaya [19].
\end{abstract}

KEY WORDS AND PHRASES: Variational inequality, nonlinear operator of hyperbolic-parabolic type, weak solutions.

AMS Subject Classification (1991): $35 L 85$

"This work was done while the author was visiting the University of Pisa, Italy, in a Post-Doctoral Program during 1996-1997. He was partially supported by CAPES Brasilia/Brasil under grant $n^{\circ}$. BEX2480-4. 


\section{INTRODUCTION}

Hyperbolic-parabolic inequalities belong to a class of inequalities of variable type, see Lar'kin, Novikov and Yanenko [22]. These inequalities are interesting not only from the point of view of the general theory of PDE, but also because of various applications in Mathematical Physics and Mechanics.

The most representative inequality of this class is the transonic Karman inequality

$$
u^{\prime} u^{\prime \prime}-u_{x x} \geq 0
$$

which models flows of a compressible gas in the transonic region, where the velocity of the gas changes from subsonic values to supersonic ones and $'=\frac{\partial}{\partial t}$. The type of the Karman inequality depends on the sign of $u^{\prime}$ : it is elliptic if $u^{\prime}<0$ parabolic if $u^{\prime}=0$, hyperbolic if $u^{\prime}>0$. In the supersonic region, including the sonic curve where $u^{\prime}=0$, the Karman inequality is hyperbolic-parabolic.

As a rule, domains in which this inequality is considered are cylindrical.

For example, the flow of a gas in the supersonic part of a Laval Nozzle which expands with $x$ can be simulated by hyperbolic-parabolic inequalities in cylindrical domains.

A great number of papers dealt with hyperbolic-parabolic equations in cylindrical domains. It seemed for us worthwhile to study this problem in the present paper.

Let $\Omega$ be a bounded open set of $R^{n}$ with smooth boundary $\Gamma$ and $T$ be an arbitrary positive real number. By $Q$ we denote the cylinder $\Omega \times(0, T)$, with lateral boundary $\sum=\Gamma \times(0, T)$. Consider the mixed problem

$$
K_{1}(x) u^{\prime \prime}+K_{2}(x) u^{\prime}-\Delta u=f \text { in } Q,
$$

where $K_{1}(x)$ and $K_{2}(x)$ are real functions defined on $\Omega$ such that

$$
\left\{K_{1}, K_{2}\right\} \in\left(L^{\infty}(\Omega)\right)^{2}, K_{1}(x) \geq 0 \text { a.e in } \Omega,
$$

and

$$
K_{2}(x) \geq \gamma>0 \text { a.e in } \Omega .
$$

Observe that on the set $\left\{x ; K_{1}(x)=0\right\}$ equation (1.1) degenerates into a parabolic equation.

This type of equation was studied by Bensoussan-Lions-Papanicolaou in [1]. Medeiros [16] studied the existence of weak solutions of the problem for (1.1) plus the nonlinear term $|u|^{\rho} u, \rho>0$. Lima [24] analyzed the equation (1.1) in a nonlinear abstract framework. In [14] Limaco studied 
(1.1) with more general non linearities $F$, where the functions $K_{1} K_{2}$ and $F$ satisfy:

$$
\begin{array}{r}
K_{1}, K_{2} \in L^{\infty}(\Omega), K_{1}(x) \geq 0 \text { and } K_{2}(x)>0 \text { a.e in } \Omega, \\
\frac{1}{K_{2}(x)} \in L^{p}(\Omega), 1<p \leq \infty,
\end{array}
$$

$F$ is a continuous such that $s F(s) \geq 0$ for all $s \in R$,

generalizing the work of Medeiros [16].

Ferreira [9] studied the problem of Limaco [14] in noncylindrical domain of $R^{n+1}$. The case when $K_{1}$ and $K_{2}$ depend also on $t \in(0, T)$ was studied by Vragov [23] and Medeiros [15]. A nonlinear problem for this case can be seen in Lar'kin [20], Maciel [2], and in Ferreira [9], [10], [11].

The study of variational inequalities was initiated by Stampacchia [7], Lions-Stampacchia [13], Brezis [8]. Cfr. also Kinderlehrer-Stampacchia [3]. In Lions [12], we can find the same type of problem for a nonlinear operator of hyperbolic, elliptic, and parabolic type but in the nondegenerate case. The degeneration of nonlinear hyperbolic equation brings essential difficulties in a case of cylindrical domains because the geometry of the domain affects the correctness of the problem. The existence and uniqueness of global and local, weak and regular solutions in cylindrical domains for other models have been treated by many authors, for example [3], [7], [13], [17], [25], [26] .

Consider the unilateral problem:

$$
\begin{aligned}
K_{1}(x) u^{\prime \prime}+K_{2}(x) u^{\prime}-\Delta u+|u|^{\rho} u & \geq f \text { in } Q, \\
u^{\prime} & \geq 0 \text { in } Q, \\
u & =0 \text { at } \sum, \\
u(x, 0)=0, u^{\prime}(x, 0) & =0, x \in \Omega,
\end{aligned}
$$

where $K_{1}(x)$ and $K_{2}(x)$ satisfy conditions $(1.2),(1.3)$ and $\rho>0$ is a real number. Lar'kin [21] analyzed the problem (1.5) in the case where $K_{1}$ and $K_{2}$ depend also on $t \in(0, T)$, the nonlinear term is of the form $\left|u^{\prime}\right|^{p} u^{\prime}$ and $u^{\prime}(x, 0) \neq 0$. Miranda and Lima [18] obtained solutions for a general unilateral problem with null initial conditions which contains (1.5) as a particular case, where $K_{1}(x)$ and $K_{2}(x)$ also satisfy the conditions (1.2), (1.3) and $\rho>0$ is a real number with some restriction.

In this paper we investigate the existence and uniqueness of the weak solutions of the mixed problem for inequality (*) with $K_{1} . K_{2}$, satisfying condition (1.4) and $F$ is a Lipschitz function satisfying the condition $s F(s) \geq 0$ 
for all $s \in R$. We get our results applying the Faedo-Galerkin method (see [12]) and using the penalty operator introduced by Lions (see [12]). This operator is different from that used by Lar'kin [21].

The paper is organized as follows:

2. Some notations and assumptions.

3. Existence of weak solutions.

4. Uniqueness.

\section{SOME NOTATIONS AND ASSUMPTIONS}

By $D(\Omega)$ we denote the space of infinitely differentiable functions with compact support contained in $\Omega$; the inner product and norm in $L^{2}(\Omega)$ and $H_{0}^{1}(\Omega)$ are denoted by $(.,),.|$.$| and ((.,)),.\|$.$\| respectively. By$ $H^{-1}(\Omega)$ we denote the dual space of $H_{0}^{1}(\Omega)$ and we let

$$
a(u, v)=\sum_{i=1}^{n} \int_{\Omega} \frac{\partial u}{\partial x_{i}} \frac{\partial v}{\partial x_{i}} d x
$$

the Dirichelet's form in $H_{0}^{1}(\Omega)$.

Let $X$ be a Banach space, we denote by $L^{p}(0, T ; X), 1 \leq p<\infty$, the Banach space of vector-valued functions $u:(0, T) \rightarrow X$ which are measurable and $\|u(t)\|_{X} \in L^{p}(0, T)$ with the norm

$$
\|u\|_{L^{p}(0, T ; X)}=\left(\int_{0}^{T}\|u(t)\|_{X}^{p} d t\right)^{\frac{1}{p}}
$$

and by $L^{\infty}(0, T ; X)$ the Banach space of vector-valued functions $u:(0, T) \rightarrow$ $X$ which are measurable and $\|u(t)\|_{X} \in L^{\infty}(0, T)$ with the norm

$$
\|u\|_{L^{\infty}(0, \infty ; X)}=e s s \sup _{0<t<T}\|u(t)\|_{X} .
$$

We consider the closed convex subset $K$ of $L^{2}(\Omega)$ defined by

$$
K=\left\{v \in L^{2}(\Omega) ; v \geq 0 \text { a.e in } \Omega\right\} \text {, with } 0 \in K .
$$

In order to use the penalty method (see Lions [12]) we define a penalty operator as follows. Let $V$ be a reflexive Banach space and $V^{\prime}$ its dual. A penalty operator is any operator $\beta: V \rightarrow V^{\prime}$ which is 

a) monotone, that is, $\langle\beta(u)-\beta(v), u-v\rangle \geq 0, \forall u, v \in V$,
b) hemicontinuous, that is, the map $\lambda \rightarrow\langle\beta(u+\lambda v), w\rangle$ is continuous,
c) $\beta(S)$ is bounded for any bounded subset $S \subset V$,
d) $\beta(u)=0 \Leftrightarrow u \in K$, where $K$ is as above.

Let the operator $\beta$ from $L^{2}(\Omega)$ into $L^{2}(\Omega), \beta=I-P$, where $P$ is the projection of $L^{2}(\Omega)$ on $K$ and

$$
\operatorname{Ker}(\beta)=\left\{u \in L^{2}(\Omega) ; \beta(u)=0\right\}=K
$$

that is, the operator $\beta$ characterizes the convex $K$.

Suppose that $K_{1}, K_{2}, F$, satisfy the following conditions:

A.1:

$$
\begin{aligned}
K_{1}(x) & \geq 0 \text { a.e in } \Omega, \\
K_{2}(x) & >0 \text { a.e in } \Omega, \\
K_{1} & \in L^{\infty}(\Omega), \\
\frac{1}{K_{2}} & \in L^{p}(\Omega), 1<p \leq \infty .
\end{aligned}
$$

A.2:

$F$ is a Lipschitz function such that $s F(s) \geq 0$ for all $s \in R$

$$
\text { where } G(s)=\int_{0}^{s} F(\xi) d \dot{\xi} \text {. }
$$

With these assumptions we shall prove our main result in this paper which is the following theorem.

\section{EXISTENCE OF WEAK SOLUTIONS}

THEOREM 3.1. Let $u_{0} \in H_{0}^{1}(\Omega) \cap H^{2}(\Omega), u_{1} \in H_{0}^{1}(\Omega) \cap K$, and $f \in$ $L^{\frac{2(p+1)}{p-1}}(Q), f^{\prime} \in L^{\frac{2(p+1)}{p-1}}(Q)$. Let assumptions A.1-A.2 hold. Then for any finite $T>0$, there exists a unique function $u$ defined in $Q$ such that

$$
\begin{gathered}
u \in L^{\infty}\left(0, T ; H_{0}^{1}(\Omega)\right) \cap L^{2}\left(0, T: H_{0}^{1}(\Omega) \cap H^{2}(\Omega)\right), \\
u^{\prime} \in L^{\frac{2 p}{p+1}}(Q), u^{\prime}(t) \in K, \forall t \in[0, T], \\
\left\{K_{1} u^{\prime}, K_{2} u^{\prime}, u^{\prime \prime}\right\} \in L^{\infty}\left(0, T ; L^{2}(\Omega)\right) \times\left\{L^{2}\left(0, T ; L^{2}(\Omega)\right)\right\}^{2} .
\end{gathered}
$$

The function $u$ satisfies the inequality 


$$
\begin{aligned}
\int_{0}^{T}\left(K_{1} u^{\prime \prime}, v-u^{\prime}\right) d t & +\int_{0}^{T}\left(K_{2} u^{\prime}, v-u^{\prime}\right) d t+\int_{0}^{T} a\left(u, v-u^{\prime}\right) d t \\
& +\int_{0}^{T}\left(F(u), v-u^{\prime}\right) d t \geq \int_{0}^{T}\left(f, v-u^{\prime}\right) d t
\end{aligned}
$$

for all $v \in L^{2}\left(0, T ; H_{0}^{1}(\Omega)\right)$ with $v(t) \in K$, a.e in $(0, T)$; and the initial conditions

$$
u(0)=u_{0}, u^{\prime}(0)=u_{1}
$$

Theorem 3.1 will be a consequence of the following.

LEMMA 3.1. Under the conditions of Theorem 3.1 for each $0<\epsilon<1$ and $\lambda>0$ there exists a unique function $u_{\epsilon \lambda}$ defined in $Q$ such that

$$
\begin{gathered}
u_{\epsilon \lambda} \in L^{\infty}\left(0, T ; H_{0}^{1}(\Omega)\right) \cap L^{2}\left(0, T ; H_{0}^{1}(\Omega) \cap H^{2}(\Omega)\right) \\
u_{\epsilon \lambda}^{\prime} \in L^{\frac{2 p}{p+1}}(Q) \\
\left\{K_{1 \epsilon} u_{\epsilon \lambda}^{\prime}, K_{2} u_{\epsilon \lambda}^{\prime}, u_{\epsilon \lambda}^{\prime \prime}\right\} \in L^{\infty}\left(0, T ; L^{2}(\Omega)\right) \times\left\{L^{2}\left(0, T ; L^{2}(\Omega)\right)\right\}^{2}
\end{gathered}
$$

where $K_{1 \epsilon}=K_{1}+\epsilon$, and

$$
\begin{gathered}
K_{1 \epsilon} u_{\epsilon \lambda}^{\prime \prime}+K_{2} u_{\epsilon \lambda}^{\prime}-\Delta u_{\epsilon \lambda}+F\left(u_{\epsilon \lambda}\right)+\frac{1}{\lambda} \beta\left(u_{\epsilon \lambda}^{\prime}\right)=f \text { in } L^{2}(Q), \\
u_{\epsilon \lambda}(0)=u_{0}, u_{\epsilon \lambda}^{\prime}(0)=u_{1} .
\end{gathered}
$$

REMARK 3.1. The same method allows to treat inequality (3.4) with the nonlinear term $\left|u^{\prime}\right|^{\rho} u^{\prime}$.

Proof. For each $\lambda>0$, we determine the equation

$$
K_{1} u_{\lambda}^{\prime \prime}+K_{2} u_{\lambda}^{\prime}-\Delta u_{\lambda}+F\left(u_{\lambda}\right)+\frac{1}{\lambda} \beta\left(u_{\lambda}^{\prime}\right)=f .
$$

The operator $\frac{1}{\lambda} \beta$ is called penalty operator. We also observe that on the set where $K_{1}(x)=0$, the equation (3.11) degenerates into a parabolic equation then, we perturbe the equation (3.11) adding the term $\epsilon u_{\lambda}^{\prime \prime}, 0<$ $\epsilon<1$, obtaining $K_{1 \epsilon}=K_{1}+\epsilon>0$ as the new coefficient of $u_{\epsilon \lambda}^{\prime \prime}$ of the 
perturbed problem (3.9)-(3.10). Moreover, the term $\frac{1}{\lambda} \beta\left(u_{\lambda}^{\prime}\right)$ permit to work in all space.

In fact, for a fixed $\epsilon \in R$ and $\lambda \in R$ with $\epsilon \in(0,1)$ and $\lambda>0$, we consider the penalized perturbed problem (3.9)-(3.10). Let $\left\{w_{\nu}\right\}_{\nu \in N}$ be a sequence of vectors of $H_{0}^{1}(\Omega) \cap H^{2}(\Omega)$ such that for each $m$, the set $\left\{w_{1}, \ldots, w_{m}\right\}$ is linearly independent and the set of finite linear combinations are dense in $H_{0}^{1}(\Omega) \cap H^{2}(\Omega)$. Let us consider the linear manifold $V_{m}=\left[w_{1}, \ldots, w_{m}\right]$, generated by the $m$ first vectors of the basis $\left\{w_{\nu}\right\}_{\nu \in N}$ and project equation (3.9) on $V_{m}$. For each $m \in N$ consider the function

$$
u_{m \varepsilon \lambda}(x, t)=\sum_{i=1}^{n} g_{m \in \lambda i}(t) w_{i}(x), 0<t \leq t_{m}, t_{m}<T,
$$

this means that we look for $u_{m \in \lambda}(t)$ in $V_{m}$, such that

$$
\begin{aligned}
& \left(K_{1 \epsilon} u_{m \epsilon \lambda}^{\prime \prime}(t), w_{i}\right)+\left(K_{2}^{\prime} u_{m \epsilon \lambda}^{\prime}(t), w_{i}\right)+a\left(u_{m \epsilon}(t), w_{i}\right)+\left(F\left(u_{m \epsilon \lambda}(t)\right), w_{i}\right) \\
& \quad+\frac{1}{\lambda}\left(\beta\left(u_{m \epsilon \lambda}^{\prime}(t)\right), w_{i}\right)=\left(f(t), w_{i}\right) \text { for all } V_{m}, 1 \leq i \leq m,
\end{aligned}
$$

$$
u_{m \in \lambda}(0)=u_{0 m} \rightarrow u_{0} \text { strongly in } H_{0}^{1}(\Omega) \cap H^{2}(\Omega),
$$

$$
u_{m \in \lambda}^{\prime}(0)=u_{1 m} \rightarrow u_{1} \text { strongly in } H_{0}^{1}(\Omega) \text {, with }\left(u_{1 m}\right)_{m \in N} \subset K \text {. }
$$

Equations (3.13) represents a system of ordinary differential equation with initial conditions (3.14). This system has solutions in $\left[0, T_{m}\right), 0<$ $T_{m}<T$ and the apriori estimates we shall obtain will permit us to extend the approximate solutions $u_{m \in \lambda}$ to the interval $[0, T]$ and also pass to the limit in $m, \epsilon$ and $\lambda, m \rightarrow \infty, \epsilon \rightarrow 0$ and $\lambda \rightarrow 0$.

Apriori estimate I. We replace $w_{i}$ in (3.13) by $2 u_{m \in \lambda}^{\prime}$ and by integrating from 0 to $t, t<T_{m}$, we have:

$$
\begin{aligned}
15) & \left|\sqrt{K_{1 \epsilon}} u_{m \epsilon \lambda}^{\prime}(t)\right|^{2}+2 \int_{0}^{t}\left|\sqrt{K_{2}} u_{m \in \lambda}^{\prime}(s)\right|^{2} d s+\left\|u_{m \epsilon \lambda}(t)\right\|^{2} \\
& +2 \int_{\Omega} G\left(u_{m \in \lambda}(x, t)\right) d x+\frac{2}{\lambda} \int_{0}^{t}\left(\beta\left(u_{m \in \lambda}^{\prime}(s), u_{m \in \lambda}^{\prime}(s)\right) d s\right. \\
= & 2 \int_{0}^{t}\left(f(s), u_{m \in \lambda}^{\prime}(s)\right) e^{i} s+\left|\sqrt{K_{1 \epsilon}} u_{1 m}\right|^{2}+\left\|u_{0 m}\right\|^{2}+\int_{\Omega} G\left(u_{0 m}(x)\right) d x .
\end{aligned}
$$

Since $F$ is Lipschitzian with Lipschitz constant $\alpha$ and $F(0)=0$, then

$$
\left|\int_{S_{i}} G\left(u_{0 m}(x)\right) d x\right|_{R} \leq \frac{\alpha}{2}\left|u_{0 m}\right|^{2} .
$$


We have

$$
\int_{0}^{t}\left(f(s), u_{m \epsilon \lambda}^{\prime}(s)\right) d s \leq C+\frac{1}{2} \int_{0}^{t}\left|\sqrt{K_{2}} u_{m \epsilon \lambda}(s)\right|^{2} d s .
$$

We know that

$$
2 \int_{\Omega} G\left(u_{m \in \lambda}(x, t)\right) d x+\frac{2}{\lambda} \int_{0}^{t}\left(\beta\left(u_{m \epsilon \lambda}^{\prime}(s), u_{m \in \lambda}^{\prime}(s)\right) d s \geq 0 .\right.
$$

Using (3.16), (3,17), (3.18), and the hypotheses on $u_{0 m}, u_{1 m}, f, K_{1}$, $K_{2}$, we obtain from $(3.15)$ the estimate

$$
\left|\sqrt{K_{1 \epsilon}} u_{m \epsilon \lambda}^{\prime}(t)\right|^{2}+\frac{3}{2} \int_{0}^{t}\left|\sqrt{K_{2}} u_{m \epsilon \lambda}(s)\right|^{2} d s+\left\|u_{m \epsilon \lambda}(t)\right\|^{2} \leq C(\alpha)
$$

where $C(\alpha)$ depends only on $\alpha$. It follows that $\left\|u_{m \in \lambda}(t)\right\|$, and therefore $\left|u_{m \epsilon \lambda}(t)\right|$, are bounded by $C$, which is sufficient to continue $u_{m \in \lambda}$ to all interval $[0, T]$.

Since $\frac{2 p}{p+1}>1$, we have

$$
\left\|u_{m \in \lambda}^{\prime}\right\|_{L^{\frac{2 p}{p+1}}(Q)} \leq C
$$

where $C$ is a positive constant independent of $m, \epsilon$ and $\lambda$.

From $(3.20)$ and the properties of $\beta$ we obtain

$$
\left|\beta\left(u_{m \in \lambda}^{\prime}\right)\right|_{L \frac{2 p}{p-1}(Q)} \leq C,
$$

Observe that estimates (3.19), (3.20) and (3.21) imply that there exists a subsequence of $\left(u_{m \in \lambda}\right)$, which we still denote by $\left(u_{m \in \lambda}\right)$, and a function $u_{\epsilon \lambda}$ such that

$$
\begin{aligned}
u_{m \epsilon \lambda} & \longrightarrow u_{\epsilon \lambda} \text { weak-star in } L^{\infty}\left(0, T ; H_{0}^{1}(\Omega)\right), \\
\sqrt{K_{1 \epsilon}} u_{m \epsilon \lambda}^{\prime} & \longrightarrow \sqrt{K_{1 \epsilon}} u_{\epsilon \lambda}^{\prime} \text { weak star in } L^{\infty}\left(0, T ; L^{2}(\Omega)\right), \\
\sqrt{K_{2}} u_{m \epsilon \lambda}^{\prime} & \longrightarrow \sqrt{K_{2}} u_{\epsilon \lambda}^{\prime} \text { weakly in } L^{2}\left(0, T \cdot L^{2}(\Omega)\right), \\
u_{m \epsilon \lambda}^{\prime} & \longrightarrow u_{\epsilon \lambda}^{\prime} \text { weakly in } L^{\frac{2 p}{p+1}}\left(0, T ; L^{\frac{2 p}{p+1}}(\Omega)\right), \\
\beta\left(u_{m \epsilon \lambda}^{\prime}\right) & \longrightarrow \beta\left(u_{\epsilon \lambda}^{\prime}\right) \text { weakly in } L^{\frac{2 p}{p-1}}\left(0, T ; L^{\frac{2 p}{p-1}}(\Omega)\right)
\end{aligned}
$$

as $m \longrightarrow \infty$. 
Let us now study the nonlinear term. From $(3.22)_{1},(3.22)_{4}$ and since the injection of $H_{0}^{1}(\Omega)$ in $L^{\frac{2 p}{p+1}}(\Omega)$ is compact, we have, after passing to a subsequence, that $u_{m \epsilon \lambda}$ convergs to $u_{\epsilon \lambda}$ almost everywhere in $Q$. Then we have

$$
F\left(u_{m \epsilon \lambda}\right) \longrightarrow F\left(u_{\epsilon \lambda}\right) \text { a.e. in } Q \text {, as } m \longrightarrow \infty .
$$

Since $F$ is Lipschitzian with constant $\alpha>0$, we obtain

$$
\begin{aligned}
& \left|F\left(u_{m \epsilon \lambda}\right)\right|_{L^{2}\left(0, T ; L^{2}(\Omega)\right)}=\left(\int_{0}^{T}\left|F\left(u_{m \in \lambda}(t)\right)\right|^{2} d t\right)^{\frac{1}{2}} \\
& \leq\left(\alpha^{2} \int_{0}^{T}\left|u_{m \in \lambda}(t)\right|^{2} d t\right)^{\frac{1}{2}}=\alpha\left|u_{m \epsilon \lambda}\right|_{L^{2}\left(0 . T ; L^{2}(\Omega)\right)} .
\end{aligned}
$$

Using (3.19) and the continuous inclusion of $L^{\infty}\left(0, T ; L^{2}(\Omega)\right)$ into $L^{2}\left(0, T ; L^{2}(\Omega)\right)$, we obtain

$$
\left|F\left(u_{m \in \lambda}\right)\right|_{L^{2}\left(0, T ; L^{2}(\Omega)\right)} \leq C(\alpha),
$$

where $C(\alpha)$ is a constant which depends only on $\alpha$.

From (3.24), (3.25) and Lions [12]. Ch.I, Lemma 1.3, we conclude that

$$
F\left(u_{m \in \lambda}\right) \longrightarrow F\left(u_{\epsilon \lambda}\right) \text { weakly in } L^{2}\left(0, T ; L^{2}(\Omega)\right),
$$

as $m \longrightarrow \infty$.

Apriori estimate II. Now, we differentiate equation (3.13) with respect to $t$ and use a result from Kinderleher and Stampacchia (see[3], p.31). Then we have

$$
\begin{aligned}
& \left(K_{1 \epsilon} u_{m \in \lambda}^{\prime \prime \prime}(t), w_{i}\right)+\left(K_{2} u_{m \in \lambda}^{\prime \prime}(t), w_{i}\right)+a\left(u_{m \in \lambda}^{\prime}(t), w_{i}\right) \\
& +\left(F^{\prime}\left(u_{m \in \lambda}(t) u_{m \in \lambda}^{\prime}(t), w_{i}\right)+\frac{1}{\lambda}\left(\left(\beta\left(u_{m \in \lambda}^{\prime}(t)\right)\right)^{\prime}, w_{i}\right)\right. \\
= & \left(f^{\prime}(t), w_{i}\right), 1 \leq i \leq m .
\end{aligned}
$$

Replacing $w_{i}$ in (3.27) by $2 g_{m \in \lambda i}^{\prime \prime}(t)$, adding from $i=1$ to $i=m$ and integrating in $[0, T] t<T$, we get

$$
+2 \int_{0}^{t}\left(F^{\prime}\left(u_{m \epsilon \lambda}(s) u_{m \in \lambda}^{\prime}(s), u_{m \in \lambda}^{\prime \prime}(s)\right) d s+\frac{2}{\lambda} \int_{0}^{t}\left(\left(\beta\left(u_{m \epsilon \lambda}^{\prime}(s)\right)\right)^{\prime}, u_{m \in \lambda}^{\prime \prime}(s)\right) d s\right.
$$




$$
=2 \int_{0}^{t}\left(f^{\prime}(s), u_{m \in \lambda}^{\prime \prime}(s)\right) d s+\left|\sqrt{K_{1 \epsilon}} u_{m \in \lambda}^{\prime \prime}(0)\right|^{2}+\left\|u_{1 m}\right\|^{2} .
$$

We have

$$
\int_{0}^{t}\left(f^{\prime}(s), u_{m \in \lambda}^{\prime \prime}(s)\right) \leq C+\frac{1}{2} \int_{0}^{t}\left|\sqrt{K_{2}} u_{m \in \lambda}^{\prime \prime}(s)\right|^{2} d s
$$

since $\beta$ is monotone. Then for $h>0$

$$
\left(\frac{\beta\left(u_{m \in \lambda}^{\prime}(s+h)-u_{m \in \lambda}^{\prime}(s)\right.}{h}, \frac{u_{m \in \lambda}^{\prime}(s+h)-u_{m \in \lambda}^{\prime}(s)}{h}\right) \geq 0,
$$

and when $h \longrightarrow 0$ we have $\frac{2}{\lambda}\left(\left(\beta\left(u_{m \in \lambda}(s)\right)\right)^{\prime}, u_{m \in \lambda}^{\prime \prime}(s)\right) \geq 0$.

The assumption that $u_{1} \in K,(3.14)_{2}$ and the properties of $\beta$ gives

$$
\left\langle\beta\left(u_{1 m}\right), u_{m \in \lambda}^{\prime \prime}(0)\right\rangle=0
$$

for $m$ large enough.

Now, we need an estimate for $u_{m \in \lambda}^{\prime \prime}(0)$. For this we put $t=0$ in (3.13), and then multiply the resulting equality by $g_{m \in \lambda i}^{\prime \prime}$, add from $i=1$ to $i=m$ and use the hypothesis about $K_{1}$ and (3.29). We obtain $\left|u_{m \in \lambda}^{\prime \prime}(0)\right| \leq C$.

Now, using that $\left|u_{m \in \lambda}^{\prime \prime}(0)\right| \leq C$, the hypothesis on $u_{1 m}, K_{1}, F,(3.27)$, (3.28), $\epsilon$, and finally estimate (3.19) it follows that

(3.32) $\left|\sqrt{K_{1}} u_{m \in \lambda}^{\prime \prime}(t)\right|^{2}+\frac{3}{2} \int_{0}^{t}\left|\sqrt{K_{2}} u_{m \in \lambda}^{\prime \prime}(s)\right|^{2} d s+\left\|u_{m \in \lambda}^{\prime}(t)\right\|^{2} \leq C$,

where $C$ is a positive constant, which is independent of $m, \epsilon, \lambda$, and $t$.

From estimate (3.32), we conclude that there exists a subsequence of $\left(u_{m \in \lambda}\right)$, which we still denote by $\left(u_{m \epsilon \lambda}\right)$, and a function $u_{\epsilon \lambda}$ such that

$$
\begin{aligned}
u_{m \in \lambda}^{\prime} & \longrightarrow u_{\epsilon \lambda}^{\prime} \text { weak-star in } L^{\infty}\left(0, T ; H_{0}^{1}(\Omega)\right) \\
\sqrt{K_{1 \epsilon}} u_{m+\lambda}^{\prime \prime} & \longrightarrow \sqrt{K_{1 \epsilon}} u_{\epsilon \lambda}^{\prime \prime} \text { weak-star in } L^{\infty}\left(0, T ; L^{2}(\Omega)\right), \\
\sqrt{K_{2}} u_{m \in \lambda}^{\prime \prime} & \longrightarrow \sqrt{K_{2}} u_{\epsilon \lambda}^{\prime \prime} \text { weakly in } L^{2}\left(0, T ; L^{2}(\Omega)\right), \\
u_{m \in \lambda}^{\prime \prime} & \longrightarrow u_{\epsilon \lambda}^{\prime \prime} \text { weakly in } L^{2}\left(0, T ; L^{2}(\Omega)\right)
\end{aligned}
$$

as $m \longrightarrow \infty$.

From the apriori estimates we see that there are functions $u_{m \in \lambda}, \lambda_{\varepsilon \lambda}$ such that we can take the limit as $m \longrightarrow \infty$ and we conclude that

$$
\begin{aligned}
\left(K_{1 \epsilon} u_{\epsilon \lambda}^{\prime \prime}(t), v\right)+ & \left(K_{2} u_{\epsilon \lambda}^{\prime}(t), v\right)+\left(-\Delta u_{\epsilon \lambda}(t), v\right)+\left(F\left(u_{\epsilon \lambda}\right), v\right) \\
+ & \frac{1}{\lambda}\left(\chi_{\epsilon \lambda}(t), v\right)=(f(t), v), \text { in } L^{2}\left(0, T ; L^{2}(\Omega)\right) .
\end{aligned}
$$


To complete the proof of Lemma 3.1 we must show that $\chi_{\epsilon \lambda}=\beta\left(u_{\epsilon \lambda}\right)$. This result can be obtained by means of the monotonicity and hemicontinuity of the operator $\beta$ (see Lions [12]). To verify the initial conditions (3.10) we use an the usual argument, as in Lions [12]. Thus the proof of Lemma 3.1 is completed.

Proof of Theorem 3.1. First we fix $\lambda \in R, \lambda>0$, observe that the estimates obtained are independent of $\epsilon$. Therefore, by the same argument used to obtain $u_{\epsilon \lambda}$ from $u_{m \epsilon \lambda}$, we can pass to the limit when $\epsilon$ goes to zero in $u_{\epsilon \lambda}$ obtaining a function $u_{\lambda}$ independent of $\epsilon$ and $\lambda$, such that

$$
\begin{aligned}
u_{\epsilon \lambda} & \longrightarrow u_{\lambda} \text { weak-star in } L^{\infty}\left(0, T ; H_{0}^{1}(\Omega)\right), \\
u_{\epsilon \lambda} & \longrightarrow u_{\lambda} \text { weakly in } L^{2}\left(0, T ; H_{0}^{1}(\Omega) \cap H^{2}(\Omega)\right), \\
u_{\epsilon \lambda}^{\prime} & \longrightarrow u_{\lambda}^{\prime} \text { weakly in } L^{\frac{2 p}{p+1}}(Q), \\
\sqrt{K_{1 \epsilon}} u_{\epsilon \lambda}^{\prime} & \longrightarrow \sqrt{K_{1}} u_{\lambda}^{\prime} \text { weak-star in } L^{\infty}\left(0, T ; L^{2}(\Omega)\right), \\
\sqrt{K_{2}} u_{\epsilon \lambda}^{\prime} & \longrightarrow \sqrt{K_{2}} u_{\lambda}^{\prime} \text { weakly in } L^{2}\left(0, T ; L^{2}(\Omega)\right), \\
\sqrt{K_{1 \epsilon}} u_{\epsilon \lambda}^{\prime \prime} & \longrightarrow \sqrt{K_{1}} u_{\lambda}^{\prime \prime} \text { weak-star in } L^{\infty}\left(0, T ; L^{2}(\Omega)\right), \\
\sqrt{K_{2}} u_{\epsilon \lambda}^{\prime \prime} & \longrightarrow \sqrt{K_{2}} u_{\lambda}^{\prime \prime} \text { weakly in } L^{2}\left(0, T ; L^{2}(\Omega)\right), \\
u_{\epsilon \lambda}^{\prime \prime} & \longrightarrow u_{\lambda}^{\prime \prime} \text { weakly in } L^{2}\left(0, T ; L^{2}(\Omega)\right), \\
F\left(u_{\epsilon \lambda}\right) & \longrightarrow F\left(u_{\lambda}\right) \text { weakly in } L^{2}\left(0, T ; L^{2}(\Omega)\right), \\
\beta\left(u_{\epsilon \lambda}^{\prime}\right) & \longrightarrow \beta\left(u_{\lambda}^{\prime}\right) \text { weakly in } L^{2}\left(0, T ; L^{2}(\Omega)\right),
\end{aligned}
$$

as $\epsilon \longrightarrow 0$. It remains to verify that $u_{\lambda}^{\prime}(t) \in K$ a.e. on $[0, T]$. From the apriori estimate I, we have

$$
\left|\beta\left(u_{\epsilon \lambda}^{\prime}\right)\right|_{L^{2}\left(0, T ; L^{2}(\Omega)\right)} \leq C \lambda, \forall \lambda>0
$$

therefore, using (3.37) and (3.36) 10 we conclude that

$$
\beta\left(u_{\lambda}^{\prime}\right)=0 \text { in } L^{2}\left(0, T ; L^{2}(\Omega)\right),
$$

which shows that $u_{\lambda}^{\prime}(t) \in K$ a.e. on $[0, T]$.

Let $v \in L^{2}\left(0, T ; H_{0}^{1}(\Omega)\right), v(t) \in K$, a.e. in $(0, T)$. Taking the limit as $\epsilon \longrightarrow 0$ in (3.35), multipling (3.35) by $v-u_{\epsilon \lambda}^{\prime}$, using (3.36) and the monotonicity of $\beta$ we obtain

$$
\begin{gathered}
\int_{0}^{T}\left(K_{1} u_{\lambda}^{\prime \prime}, v-u_{\lambda}^{\prime}\right) d t+\int_{0}^{T}\left(K_{2} u_{\lambda}^{\prime}, v-u_{\lambda}^{\prime}\right) d t+\int_{0}^{T} a\left(u_{\lambda}, v-u_{\lambda}^{\prime}\right) d t \\
+\int_{0}^{T}\left(F\left(u_{\lambda}\right), v-u_{\lambda}^{\prime}\right) d t-\int_{0}^{T}\left(f, v-u_{\lambda}^{\prime}\right) d t=
\end{gathered}
$$




$$
-\frac{1}{\lambda} \int_{0}^{T}\left(\beta\left(u_{\lambda}^{\prime}\right), v-u_{\lambda}^{\prime}\right) d t=-\frac{1}{\lambda} \int_{0}^{T}\left(\beta\left(u_{\lambda}^{\prime}\right)-\beta(v), v-u_{\lambda}^{\prime}\right) d t \geq 0 .
$$

Observe that all estimates obtained are independent of $\lambda$. Therefore by the same argument used above we can pass to the limit in $u_{\lambda}$ (or a subsequence) when $\lambda$ goes to zero obtaining a function $u$ independent of $\lambda$, satisfying the convergences (3.36) and (3.38).

Taking the limit when $\lambda \longrightarrow 0$ in (3.39) we obtain the inequality (3.4) and the limit $u$ obtained above satisfies the conditions (3.1)-(3.3) of Theorem 3.1. To verify the initial conditions (3.5) we use an argument as in Lions [12]. Thus the proof of Theorem 3.1 is completed.

\section{UNIQUENESS}

We do not prove uniqueness for the general case. For some particular $F$ we can use Ladyzenskaya's methods (see [19]) to obtain the uniqueness of solutions. So we have uniqueness in the following cases.

Case 1. If $n=1$ and $F$ is local Lipschitz function.

Case 2. If $n \geq 2, F \in C^{1}(R),|F(s)| \leq C|s|^{\rho}, \forall s \in R$, where $0<\rho \leq$ $\frac{2}{n-2}$ if $n>2$ or $0<\rho<\infty$ if $n=2$.

Case 3. If $F$ is a global Lipschitz function.

REMARK 4.1. Results of Theorem 3.1 can be easily generalized for more general inequalities

$$
K_{1}(x, t) u_{t t}+K_{2}(x, t) u_{t}+A(t) u+H(u) \geq f(x, t),
$$

where $A(t)$ is a strictly elliptic operator and a continuous function $H(u)$ satisfies the condition $s H(s) \geq 0$. 


\section{ACKNOWLEDGEMENT}

The author would like to express his sincere gratitude to Professor Hugo Beirão da Veiga for his invitation to visit the University of Pisa in a PostDoctoral Program during 1996-1997 and for his hospitality. He is also very grateful to Professor Ricardo Soto Montero for attention to my paper and to the referee of the Proyecciones Revista de Matematicas for his valuable suggestions which improved this paper. Finally, the author wishes to thank Professor Waldemar D. Bastos for the previous important conversations on the topics related with this work.

\section{References}

[1] Bensoussan, A.and Lions, J. L. and Papanicolaou, G., Pertubations et augmentation des conditions initiales, in: Singular Pertubation and Boundary Layer Theory, Springer-Velarg, Lyon., pp. 10-26, (1976).

[2] Maciel, A. B., On a hyperbolic-parabolic equation with a continuous nonlinearity, Nonlinear Analysis T. M. A., 20, pp. 745-754, (1993).

[3] Kinderlehrer, D. and Stampacchia, G., An Introduction to Variational Inequalities and their Applications, Academic Press, New York, (1980).

[4] Pereira, D. C., Existence, uniqueness and asymptotic behavior for solutions of the nonlinear beam equation, Nonlinear Analysis T. M. A., 14, pp. 613-623, (1990).

[5] Browder, F. E., Nonlinear monotone operators and convex sets in Banach spaces. Bull. Am. Math. Society., 71, pp. 780-785, (1965).

[6] Menzala, G. P., On global classical solutions of a nonlinear wave equation, Applicable Analysis., 10, pp. 179-195, (1980).

[7] Stampacchia, G., Formes bilineaires sur les ensembles convexes. C.R. Acad. Sc. Paris., 258, pp. 4413-4416, (1964). 
[8] Brezis, H., Problèmes unilatereaux. J. Math. Pures et Appl., 51, pp. $1-168,(1972)$.

[9] Ferreira, J., Nonlinear hyperbolic-parabolic partial differential equation in noncylindrical domain, Rendiconti del Circolo Matematico di Palermo., 44, pp. 135-146, (1995).

[10] Ferreira, J., On weak solutions of a nonlinear hyperbolic-parabolic partial differential equation. Comp. Appl. Math., 14, No 3, pp. 1-15, (1995).

[11] Ferreira, J., On weak solutions of semilinear hyperbolic-parabolic equations. International Journal of Mathematics and Mathematical Sciences., 19, $\mathrm{n}^{\circ} .4$, pp. 751-758, (1996).

[12] Lions, J. L., Quelques méthodes des résolutions des problèm aux limites nonlinéaires, Dunod, Paris, (1969).

[13] Lions, J. L. and Stampacchis, G., Variational inequalities. Com. Pure and Appl., Math. 20, pp. 493-519 (1967).

[14] Limaco, J., On weak solutions of a nonlinear hyperbolic-parabolic equations. Preprint.

[15] Medeiros, L. A., L.A. Remarks on hyperbolic-parabolic partial differential equations, Memórias de Matemática. 123, IM-UFRJ, (1981).

[16] Medeiros, L. A., L.A. Nonlinear hyperbolic-parabolic partial differential equations. Funcialaj Ekvacioj., 23, No 2, pp. 151-158, (1980).

[17] Medeiros, L. A. and Miranda, M. M., Local solutions for a nonlinear unilateral problem, Rev. Roumaine Math. Pures Appl., 31, pp. 371$382,(1986)$.

[18] Miranda, M. M. and Lima, O. A., One-sided problem for a nonlinear hyperbolic-parabolic equation, Proceedings of IX CNMAC., Brasilia, (1986).

[19] Visik, M. and Ladayzenskaya, O., On boundary value problem for and certais class of operators equations, Amer. Math. Soc. Transl., 2 $\mathrm{N}^{\circ} .10,(1958)$.

[20] Lar'kin, N. A., N.A. Boundary problem in the large for a class of hyperbolic-parabolic equation, Sib. Math. J., 18 No 6, pp. 1003-1006, (1977). 
[21] Lar'kin, N. A., Inequalities for degenerating hyperbolic operators, Sib. Math. J., 21, No 4, pp. 613-617, (1980).

[22] Lar'kin, N. A. and Novikov, V. A. and Yanenko, N. N., Towards a Theory of Variable-Type Equations, in: Numerical Methods in Fluid Dynamics. (N.N. Yanenko and Yu. I. Shokiu Editores), Moscow, Mir., pp. 315-335, (1984).

[23] Vragov, N., On a mixed problem of a hyperbolic-parabolic equations, Dokl. Akad. Nauk. USSR., $224 \mathrm{~N}^{\circ}$ 2, pp. 1179-1183, (1975).

[24] Lima, O. A., Existence and uniqueness of solutions for an abstract nonlinear hyperbolic-parabolic equation, Applicable Analysis., 24, pp. $101-106,(1987)$.

[25] Ebihara, Y., Modified variational inequalities to semilinear wave equations. Nonlinear Analysis., 7, No 8, pp. 821-826, (1983).

[26] Ebihara, Y. and Medeiros, L. A. and Miranda, M. M., On a variational inequality for a nonlinear operator of hyperbolic type, Bol. Soc. Mat. 16, pp. 41-54, (1985).

Received: December 27, 1996.

\section{Jorge Ferreira}

Departamento de Matemáticas

Universidad Estadual de Maringá

Av. Colombo 5790

Campus Universitario

Zona 07

Maringá - Paraná

Brasil 